\title{
Transdiagnostic Patterns of Sensory Processing in Autism and ADHD
}

Nichole E. Scheerer, Anahid Pourtousi, Connie Yang, Zining Ding, Bobby Stojanoski, Evdokia Anagnostou, Robert Nicolson, Elizabeth Kelley, Stelios Georgiades, Jennifer Crosbie, Russell Schachar, Muhammad Ayub, Ryan A. Stevenson

\section{Affiliations}

NS - Western University, Brain and Mind Institute, 1151 Richmond St, London, ON, Canada, N6A 3K7, nikkischeerer@gmail.com, *Corresponding Author

CY, AP, ZD - Western University, Department of Psychology, 1151 Richmond St, London, ON, Canada, N6A3K7, apourto@uwo.ca, cyang393@uwo.ca, zding52@uwo.ca

BS - Western University, Brain and Mind Institute, 1151 Richmond St, London, ON, Canada, N6A 3K7; University of Ontario Institute of Technology, Faculty of Social Science and Humanities, 2000 Simcoe Street North, Oshawa, ON L1H 7K4, Canada, bobby.stojanoski@ontariotechu.ca

EA - Holland Bloorview Kids Rehabilitation Hospital, 150 Kilgour Rd, East York, ON M4G1R8, eanagnostou@hollandbloorview.ca

RN - Western University, Department of Psychiatry, 800 Commissioners Road East, B8-026, London, ON. N6A 5W9, Rnicolso@uwo.ca 
EK - Queens University, Department of Psychology, 62 Arch St., Kingston, ON

K7L 3N6; Queens University, Department of Psychiatry, 62 Arch St., Kingston, ON

K7L 3N6, kelleyb@queensu.ca

SG - McMaster University, 1280 Main St W, Hamilton, ON L8S 4L8, georgis@mcmaster.ca

JC - University of Toronto, Department of Psychiatry, The Hospital for Sick Children, 555

University Ave, Toronto, Ontario, M5G 1X8, jennifer.crosbie@sickkids.ca.

RS - University of Toronto, Department of Psychiatry, The Hospital for Sick Children, 555

University Ave, Toronto, Ontario, M5G 1X8, russell.schachar@sickkids.ca.

MA - Department of Psychiatry, 62 Arch St., Kingston, ON, K7L 3N6, ma84@queensu.ca

RS - Western University, Department of Psychology, 1151 Richmond St, London, ON,

Canada, N6A 3K7

Western University, Brain and Mind Institute, 1151 Richmond St, London, ON, Canada, N6A 3K7

Western University, Department of Psychiatry, 1151 Richmond St, London, ON, Canada, N6A3K7, rsteve28@uwo.ca 


\section{Abstract (120 Word Max)}

Sensory processing abilities are highly variable within and across people diagnosed with autism spectrum disorder (ASD) and attention-deficit/hyperactivity disorder (ADHD). This study examined the transdiagnostic nature of sensory processing abilities, and their association with features of ASD and ADHD, in a large sample of autistic people $(n=495)$ and people with ADHD $(n=461)$. Five similar data-driven sensory phenotypes characterized sensory processing abilities, and showed similar patterns of association with features of ASD and ADHD, across both diagnostic groups. These results demonstrate the transdiagnostic nature of sensory processing abilities, while contributing to a growing body of literature that suggests the ASD and ADHD diagnostic labels have poor explanatory power.

\section{Keywords}

Sensory Phenotypes, Cluster Analysis, Autism Spectrum Disorder, Attention Deficit Hyperactivity Disorder, Social Communication, Restrictive and Repetitive Behaviours,

\section{Introduction}

Autism spectrum disorder (ASD) and attention-deficit/hyperactivity disorder (ADHD) are complex neurodevelopmental conditions that both demonstrate large within-disorder heterogeneity. Autism is characterized by persistent deficits in social communication and interaction and restricted, repetitive patterns of behaviour, interests, or activities, while ADHD is characterized by a persistent pattern of inattention and/or hyperactivity-impulsivity that interferes with functioning or development (APA, 2013). Despite their distinct diagnostic criteria, ASD and ADHD demonstrate a high degree of between group overlap (Baribeau, 2015; Brierley et al., 
2021; Demopoulos et al., 2013; Jacobs et al., 2021; Krakowski et al., 2020; Kushki et al., 2019, 2021). Further, ASD and ADHD are highly comorbid, with rates of ADHD among autistic people reported to range from 25.7-65\% (Hossain et al., 2020).

In both autistic people and people with ADHD, differences in sensory processing have been well documented. As a whole, the autistic (Ben-Sasson et al., 2009; Lane et al., 2010, 2011, 2014; Scheerer et al., 2021; Tomchek \& Dunn, 2007) and ADHD (Mangeot et al., 2001; Pfeiffer et al., 2015) populations consistently demonstrate sensory processing differences across all sensory domains. Further, these sensory differences are highly variable showing large withindisorder heterogeneity (ASD: Lane et al., 2010, 2011, 2014; Scheerer et al., 2010; ADHD: Little et al., 2017; Mangeot et al., 2001).

While a breadth of studies demonstrate sensory processing differences in autistic people or people with ADHD, few studies have compared sensory processing across these diagnostic groups. Autistic children and children with ADHD show similarities in sensory processing, according to the Child Sensory Profile 2 (SP2; Dunn, 2014), with both diagnostic groups differing from typically developing children (Little et al., 2018). Given the similarities in sensory processing across diagnostic groups, coupled with the high degree of heterogeneity in sensory processing patterns within each of these diagnostic groups, researchers have hypothesized that there may be a transdiagnostic underlying sensory differences across diagnoses. For this reason, a more focused investigation of overlapping sensory processing patterns in these diagnostic groups is warranted.

Clustering techniques represent an ideal solution for parsing such sensory heterogeneity. Clustering involves grouping individuals with similar sensory processing abilities together in 
such a way that individuals in the same cluster have more similar sensory processing abilities to each other than individuals in other clusters. These resultant clusters can be thought of as sensory phenotypes, or distinct patterns of sensory processing abilities that commonly co-occur together.

Using this clustering technique to explore sensory processing patterns in autistic people commonly yields between three- and five-cluster solutions (Dwyer et al., 2020; Lane et al., 2010, 2011, 2014; Scheerer et al., 2021; Uljarevic et al., 2016). Across these studies, a sensory adaptive phenotype has been identified that describes autistic people with mostly typical sensory processing. A generalized sensory differences phenotype also emerges, describing autistic people who have difficulties across all sensory domains (Dwyer et al., 2020; Lane et al., 2010, 2011, 2014; Scheerer et al., 2021; Uljarevic et al., 2016). While less consistently reported, other intermediate phenotypes exhibiting distinct patterns of sensory differences in autistic people have been identified including a sensory moderate phenotype (Uljarevic et al., 2016), a taste and smell sensitivity phenotypes (Lane et al., 2010, 2011, 2014; Scheerer et al., 2021), an underresponsive and sensory seeking phenotype (Lane et al., 2011; Scheerer et al., 2021), a tactile and movement difficulties phenotype (Lane et al., 2011), and a movement difficulties with low energy phenotype (Lane et al., 2014; Scheerer et al., 2021).

While clustering techniques have helped to parse sensory heterogeneity in autistic people, this area is unstudied in ADHD, to our knowledge. If sensory phenotypes are indeed transdiagnostic, we would expect to see an ADHD cohort cluster into sensory phenotypes that resembled those in autism. If, however, sensory processing associated with ASD and ADHD are specific to their diagnostic constructs, then diagnosis-specific phenotypes should emerge. With that said, it is unclear whether sensory processing differences in ADHD will cluster into discrete 
phenotypes at all. If no meaningful sensory phenotypes emerge within the ADHD group, this will provide evidence against the hypothesis of sensory phenotypes being transdiagnostic.

An important aspect of sensory phenotypes as a clinically meaningful way of parsing heterogeneity is that discrete phenotypes differentially relate to traits such as clinical profiles, cognitive ability, and demographic factors. In autism, these sensory phenotypes have been linked with behavioural traits such as adaptive functioning (Lane et al., 2010; Tillmann et al., 2020; Scheerer et al., 2021), autistic traits (Lane et al., 2010; Tillmann et al., 2020; Scheerer et al., 2021), and ADHD traits (Lai et al., 2019; Tillmann et al., 2020; Scheerer et al., 2021). Given that sensory phenotypes have not been investigated in ADHD, it follows that the relationships between discrete phenotypes and other clinically meaningful factors is unknown. If sensory phenotypes are transdiagnostic, we would predict that the pattern of other clinically-relevant traits across sensory phenotypes would be equivalent across diagnostic groups, even where overall differences in the levels of these traits may be observed (for example, possibly higher levels of cognitive ability in ADHD).

The primary aim of the current study is to examine the transdiagnostic nature of sensory processing abilities in a large sample of autistic people and people with ADHD. Given the substantial between group overlap in the phenotypes of autistic people and people with ADHD (Baribeau, 2015; Brierly et al., 2021; Demopoulos et al., 2013; Kushki et al., 2019, 2021), we expect sensory abilities will cluster into similar phenotypes across these diagnostic groups. While this will highlight the transdiagnostic nature of sensory processing abilities, this will also demonstrate that the sensory processing abilities of people with ADHD can be clustered into discrete phenotypes. Given sensory abilities have been shown to be predictive of behavioural traits, we expect the resultant sensory phenotypes will differentially relate to the core diagnostic 
features of ASD and ADHD. Together these findings will help to disambiguate the relationship between the core features of ASD and ADHD that are often obscured by strikingly large within and between group heterogeneity.

\section{Methods}

\section{Participants}

495 autistic people and 461 people with ADHD were included in this study (see Table 1 for detailed participant characteristics). Participants in the ASD were aged 1-21 ( $M_{\text {age }}=9.21, S D$ $=4.54)$ and were $76.8 \%$ male. The ADHD group were aged 3-18 $\left(M_{\text {age }}=9.59, S D=2.82\right)$ and were $71.4 \%$ male. Participant data was extracted from the Province of Ontario Neurodevelopmental Disorder (POND) Network's database (https://pond-network.ca/). Participants were included if their parent or caregiver had completed the Short Sensory Profile (SSP; McIntosh et al., 1999) and they had a diagnosis of either ADHD or autism, including ASD, Autism, Asperger's, or Pervasive Developmental Disorder Not Otherwise Specified. Diagnoses were made by general and pediatric physicians, psychiatrists, developmental behavioural pediatricians, and psychologists. For ASD, diagnoses were confirmed using the Autism Diagnostic Observation Schedule (ADOS; Gotham et al., 2007) and Autism Diagnostic Interview (ADI-R; Lord et al., 1994), while ADHD diagnoses were confirmed by the Parent Interview for Child Symptoms (Ickowicz et al., 2006), administered by reliable examiners. Given an experimental aim was to compare sensory processing across ASD and ADHD, participants were excluded if they had an ASD and ADHD. However, individuals with other comorbid diagnoses were not excluded given ASD and ADHD have significant diagnostic overlap with other diagnoses (Hossain et al., 2020). Common comorbidities included anxiety disorders 
(11.31\% ASD, 25.38\% ADHD), intellectual disabilities (9.29\% ASD, 27.33\% ADHD), and learning disorders $(6.06 \%$ ASD, $26.46 \%$ ADHD). Participants and their parents or caregivers also completed a range of measures to assess the participant's IQ, sensory processing abilities, ASD traits, ADHD traits, and OCD traits (see Table 1). Study procedures were approved by the Research Ethics Board at Western University, and ethical approval was also obtained at each data collection site, in accordance with the World Medical Association's 2013 Declaration of Helsinki. The present study has been pre-registered with the Open Science Framework (https://osf.io/fnv5m/).

\section{Materials}

IQ was tested using measures of intelligence that were appropriate for the participant's age and developmental level. Weschler tests, the Wechsler Abbreviated Scales of Intelligence First Edition (Wechsler, 1999; $\mathrm{n}=1$ ), Second Edition (Weschler, 2011); $\mathrm{n}=430$ ), the Wechsler Intelligence Scale for Children Version 4 (Weschler, 2003; $\mathrm{n}=45$ ) and Version 5 (Wechsler, 2014); $\mathrm{n}=75$ ), and the Wechsler Preschool and Primary Scale of Intelligence Version 4 (Weschler, 2012; $\mathrm{n}=3$ ), were prioritized when children were of the appropriate age, were verbally fluent, and there was sufficient time. The Stanford-Binet Intelligence Scale (Roid \& Pomplun, 2012; $n=114$ ), the Mullen Scales of Early Learning (Mullen, 1995; $n=42$ ), and the Leiter International Performance Scale Version 3 (Roid et al., 2013; n =6) were used for those who were too young or unable to complete the Weschler tests. IQ data for 240 participants were not available.

Short Sensory Profile. The Short Sensory Profile (SSP; McIntosh 1999) is a wellvalidated parent-report questionnaire that assesses daily behaviours associated with abnormal 
responses to sensory stimuli in children aged 3-10 years. A total of 38 items are categorized into one of seven sensory domains: Tactile Sensitivity (7 items), Taste/Smell Sensitivity (4 items), Movement Sensitivity (3 items), Underresponsive/Seeks Sensation (7 items), Auditory Filtering (6 items) Low Energy/Weak (6 items), and Visual/Auditory Sensitivity (5 items). Parents respond to each question on a five-point Likert scale (always $(100 \%$ of the time $)=1$, frequently $(75 \%$ of the time $)=2$, occasionally $(50 \%$ of the time $)=3$, seldom $(25 \%$ of the time $)=4$, or never $(0 \%$ of the time $)=5)$ indicating the frequency with which their child displays the sensory behavior. The SSP produces a raw score with lower scores indicate greater sensory processing abnormalities. The SSP has been shown to have strong internal consistency in individuals with ASD (e.g. .89; Tomchek et al., 2014, .92; Scheerer et al., 2021) and is widely used in studies of sensory perception as it covers a wide range of sensory processing domains. While the SSP was initially developed on typically developing children, a confirmatory factory analysis has indicated that the seven-subscale structure is also appropriate for quantifying sensory processing in children and young adults diagnosed with autism or ADHD between the ages of 1 and 22 years (Parks et al., 2020).

Repetitive Behavior Scale - Revised (RBS-R). The RBS-R (Lam \& Aman, 2007) is a 43-item questionnaire administered to parents of children ages 6-17. The RBS-R aims to measure the breadth of repetitive behaviors in children and adolescents with ASD. Items are scored as 0Behavior does not occur, 1-Behavior occurs and is a mild problem, 2-Behavior occurs and is a moderate problem, 3-Behavior occurs and is a severe problem. The RBS-R produces a raw score, with total overall scores indicating the prevalence of more problematic behaviors. We assessed repetitive behaviors using a four-factor structure consisting of Stereotypy, Self-Injury, Compulsions, and Ritualistic/Sameness subscales (Brierley et al., 2021). Cronbach's alpha for 
these subscales indicates high internal consistency with alphas ranging from $0.8-0.92$ (Brierley et al., 2021).

Social Communication Questionnaire. The Social Communication Questionnaire Lifetime Form (SCQ; Rutter, et al., 2003) is a standardized parent-report screening questionnaire to evaluate communications skills and social functioning in individuals aged 4-40 years who are believed to be autistic. The SCQ considers lifetime characteristics across 40 items that measure three domains: Social Relating, Communication, and Range of Interests that are assessed using yes/no responses. The SCQ produces a total raw score that is calculated by summing all items. A total score of 15 or greater suggests that the individual is likely to be on the autism spectrum. The SCQ has high internal consistency (Cronbach's alpha =0.87; (Rutter, et al., 2003), and good discriminative validity when distinguishing between children with ASD and non-ASD diagnoses. The sensitivity of the SCQ is about $96 \%$, while the specificity is about $80 \%$, in samples of children without intellectual disability (Rutter, et al., 2003). Note that while the SCQ contains questions pertaining to a child's range of interests, given the questions are primarily social in nature, the SCQ was used as an index of autistic social behaviours for this study.

\section{Strengths and Weaknesses of Attention-Deficit/Hyperactivity Disorder Symptoms of} Normal Behavior Scale (SWAN). The SWAN (Arnett et al., 2013) is an 18-item caregiver questionnaire designed for children under the age of 18 years. The questionnaire includes scoring of both strengths and weaknesses associated with symptoms of ADHD. Each question is scored on a seven-point scale, with Far Below Average =3, Below Average = 2, Somewhat Below Average $=1$, Average $=0$, Somewhat Above Average $=-1$, Above Average $=-2$, and Far Above Average $=-3$. The SWAN produces a raw score, with higher scores indicate greater symptomatology. Two raw subscale scores can be produced, the inattention subscale, and the 
hyperactivity subscale. The SWAN has high internal consistency (Cronbach's $\alpha=0.88$ ), and reliability ranged from .72 to $.90(\mathrm{M}=0.82$; (Arnett et al., 2013)).

\section{Statistical Analyses}

Statistical analyses were conducted using R (v. 4.0.2, Vienna, Austria) and Jamovi (v. 1.6, Sydney, Australia). Descriptive statistics were calculated for each of the measures for the ASD and ADHD samples. The SSP subscale scores were then converted to z-scores and submitted to k-means cluster analyses to identify patterns of sensory processing separately for the ASD and ADHD samples, as well as for a combined ASD and ADHD sample. A cluster analysis is an exploratory data analysis technique used to identify subgroups (or clusters) in a dataset that represent data points that are similar to one another, yet distinct from data points in other clusters. The k-means algorithm clusters the data into a number, $\mathrm{k}$, of predefined, distinct, and non-overlapping groups where each data point only belongs to one group. Data points are assigned to a particular cluster in such a way that the sum of the squared distance between all of the data points, and the mean of all the data points that belong to that cluster, is minimized (Hartigan et al., 1979). Applying the k-means approach to the subscales of the SSP allowed us to examine how sensory processing differences cluster together, with each of the resulting clusters representing a distinct sensory phenotype. Based on a systematic review indicating 3-5 sensory phenotypes in autism, we tested k's of 2-6 (DeBoth \& Reynolds, 2017). To determine the best fit model, we used Bayesian Information Criteria (BIC; (Zhong \& Ghosh, 2003)), previous literature (Ausderau et al., 2014; Ben-Sasson et al., 2009; Lane et al., 2010, 2011, 2014; Liss et al., 2006; Little et al., 2017; Scheerer et al., 2021; Simpson et al., 2019; Uljarevic et al., 2016), and comparisons with behavioural clinical measures to help quantify the practical, real-world significance of these sensory phenotypes. Welch's one-way analysis of variances (ANOVAs) 
assuming unequal variances with follow-up Games-Howell post hoc comparisons were used to compare SSP subscale scores across the sensory clusters. Chi-square tests were used to compare sex at birth across the phenotypes for each diagnostic group. Welch's ANOVAs with GamesHowell post hoc comparisons were used to compare IQ, ASD traits, ADHD traits, and OCD traits across the clusters, separately for each diagnostic group, as well as across diagnostic groups, and their interaction. For the combined analysis, the proportion of each diagnostic group in each cluster was calculated. Given the large sample size utilized in this study, a priori effect sizes of $\eta_{\mathrm{p}}{ }^{2}=.060, w^{2}=0.06$, Cohen's $\mathrm{d}=0.5$, and Cramer's $V=.400$, or moderate, were set for all statistical analyses as indicators of meaningful effects. P-values will be reported for thoroughness, but conclusions will be drawn based on effect sizes.

\section{Results}

Table 1 reports the descriptive results for key demographic and experimental measures for both the autistic and ADHD samples. For the autistic children, mean scores on the taste/smell, movement, and visual auditory sensitivity subscales fell into the probable difference, while scores on the tactile, underresponsive/seeks sensation, auditory filtering, and low energy subscales fell into the definite difference in sensory processing function range when comparing the mean scores to normative data based on the performance of children without disabilities $(n=$ 1037; (McIntosh et al., 1999). For the children with ADHD, mean scores on the taste/smell, movement, and visual/auditory subscales fell into the typical performance range, scores on the tactile and low energy subscales fell into the probable difference range, and scores on the under responsive/ seeks sensation and auditory filtering subscales fell into the definite difference range when comparing the mean scores to normative data. Comparing across the samples, autistic individuals had more sensory processing difficulties across all subscales $(\mathrm{p}<.001)$, except for 
the under responsive/seeks sensation subscale that showed no group differences, and the auditory filtering subscale where ADHD individuals showed more sensory difficulties (see Table 1).

Table 1

Participant Characteristics

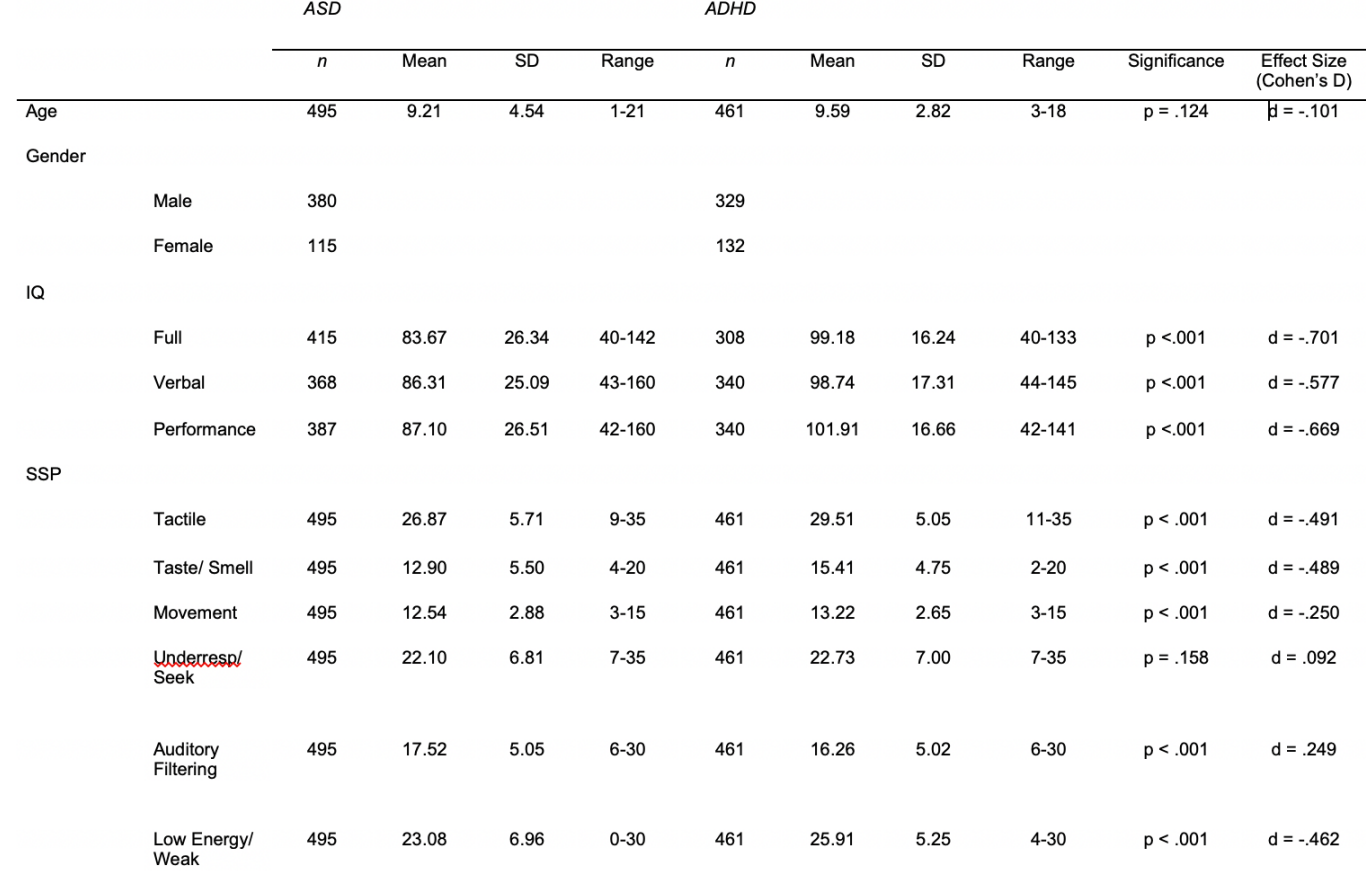

\begin{tabular}{|c|c|c|c|c|c|c|c|c|c|c|c|}
\hline & $\begin{array}{l}\text { Visual/ } \\
\text { Auditory }\end{array}$ & 495 & 17.35 & 5.16 & $5-25$ & 461 & 20.61 & 4.58 & $6-25$ & $p<.001$ & $d=-.670$ \\
\hline \multicolumn{2}{|l|}{ SCQ } & 436 & 19.54 & 7.35 & $1-37$ & 441 & 7.38 & 6.00 & $0-32$ & $p<.001$ & $d=1.812$ \\
\hline \multicolumn{12}{|c|}{ RBS-R } \\
\hline & Total & 495 & 26.72 & 20.01 & $0-92$ & 461 & 15.19 & 15.37 & $0-84$ & $p<.001$ & $d=.647$ \\
\hline & Self Iniurx & 495 & 2.59 & 3.33 & $0-18$ & 461 & 1.58 & 2.66 & $0-22$ & $p<.001$ & $\mathrm{~d}=.334$ \\
\hline & Stereotypy & 495 & 5.96 & 4.82 & $0-22$ & 461 & 2.95 & 3.88 & $0-22$ & $p<.001$ & $d=.690$ \\
\hline & $\begin{array}{l}\text { Ritualistic/ } \\
\text { Sameness }\end{array}$ & 495 & 12.72 & 9.81 & $0-43$ & 461 & 7.45 & 7.55 & $0-39$ & $p<.001$ & $d=.602$ \\
\hline & Compulsions & 495 & 3.89 & 4.39 & $0-24$ & 461 & 1.97 & 3.03 & $0-19$ & $\mathrm{p}<.001$ & $d=.509$ \\
\hline \multicolumn{12}{|l|}{ SWAN } \\
\hline & $\begin{array}{l}\text { ADHD } \\
\text { Inattentive }\end{array}$ & 369 & 4.24 & 3.04 & $0-9$ & 443 & 5.64 & 2.86 & $0-9$ & $\mathrm{p}<.001$ & $\mathrm{~d}=-.474$ \\
\hline & $\begin{array}{l}\text { ADHD } \\
\text { Hyperactive }\end{array}$ & 369 & 3.57 & 3.16 & $0-9$ & 443 & 4.37 & 3.19 & $0-9$ & $p<.001$ & $d=-.251$ \\
\hline
\end{tabular}

Abbreviation: SSP, Short Sensory Profile; IQ, Intelligence Quotient; RBS-R, - Repetitive Behaviour Scale - Revised; SCQ, Social Communication Questionnaire; SWAN, Strengths and Weaknesses of Attention-Deficit/Hyperactivity Disorder Symptoms and Normal Bebaviour Scale; ADHD I, Attention-Deficit/Hyperactivity Disorder Inattentive Scale; ADHD HI, Attention-Deficit/Hyperactivity Disorder Hyperactivity Scale

\section{Patterns of Sensory Behavior}


Results of the k-means cluster analyses conducted in R separately for both autistic children and children with ADHD's SSP data indicated that a five-cluster solution produced the best-fit model after considering previous literature, BIC values, and the practical, real-world significance of the resultant sensory phenotypes. A bootstrapping technique was used to produce 100 iterations of the five-cluster solution for each group to ensure the reliability of the selected model and BIC values were examined (see Supplemental Materials A). For both diagnostic groups, starting with a $\mathrm{K}$ of 2, the k-means cluster analysis fit a model that clustered participants by high or low sensory processing differences (see Figure $1 \& 2$ ). With the addition of each successive cluster, the model produced a group of clusters that highlighted distinct patterns of sensory processing differences. However, once the six-cluster model emerged, the new cluster failed to produce a highly differentiated pattern of sensory processing differences. Given the pattern of the SSP subscale scores across the clusters in the five-cluster model, we classified cluster 1 as a Sensory Adaptive (SA) phenotype, cluster 2 as a Generalized Sensory Differences (GSD) phenotype, cluster 3 as a Taste and Smell Sensitivity (TSS) phenotype, cluster 4 as an Underresponsive and Sensory Seeking (URSS) phenotype, and cluster 5 as a Low Energy with Weakness (LEW) phenotype (see Figure 1 and 2). 


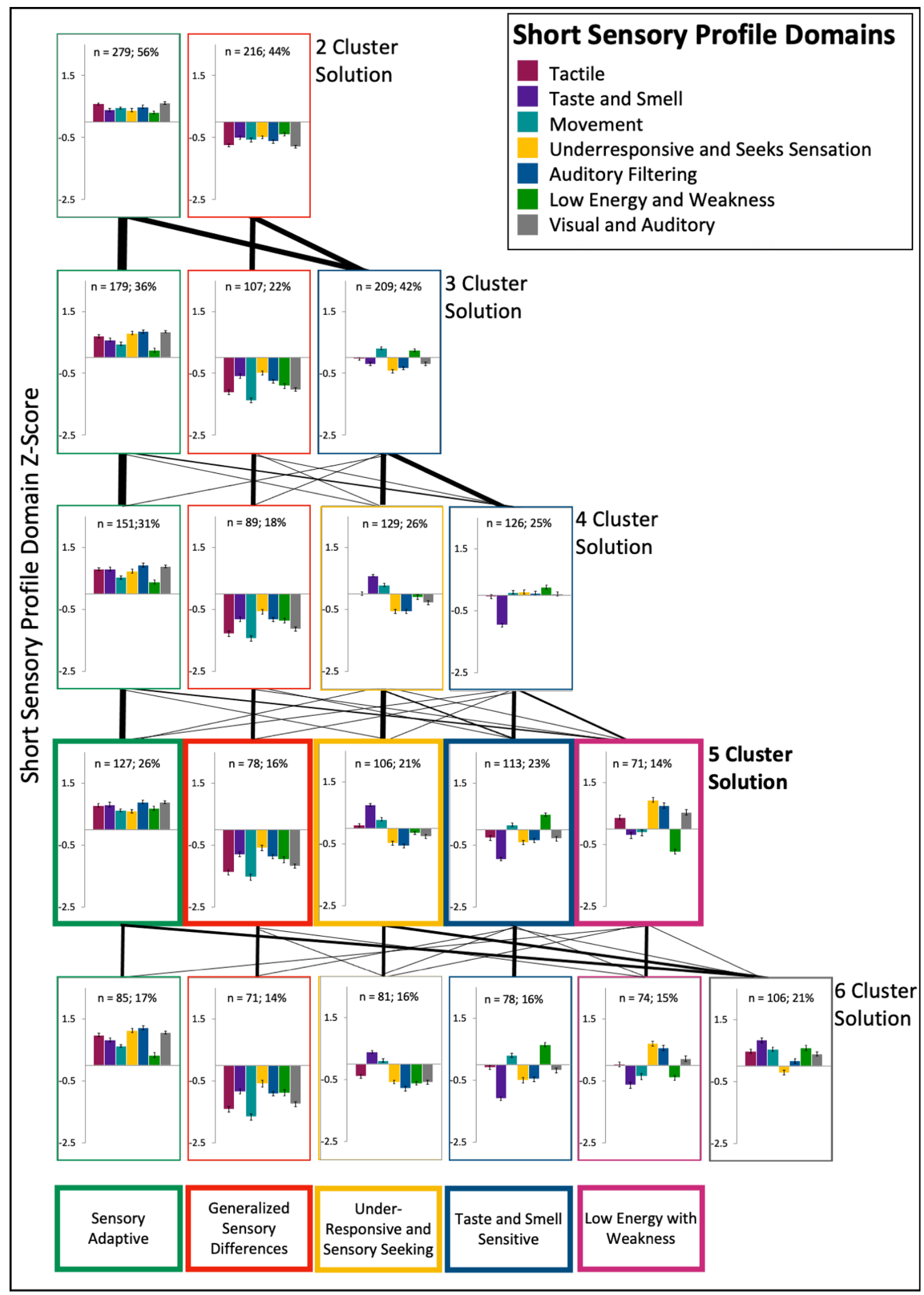


Figure 1: Short Sensory Profile Domain Z-Scores across the k 2 to 6 cluster solutions for the ASD sample. Negative z-scores are indicative of increased sensory difficulties. Line weights between cluster solutions represent the number of participants remaining/changing clusters across solutions. Error bars indicate standard error of the mean. 


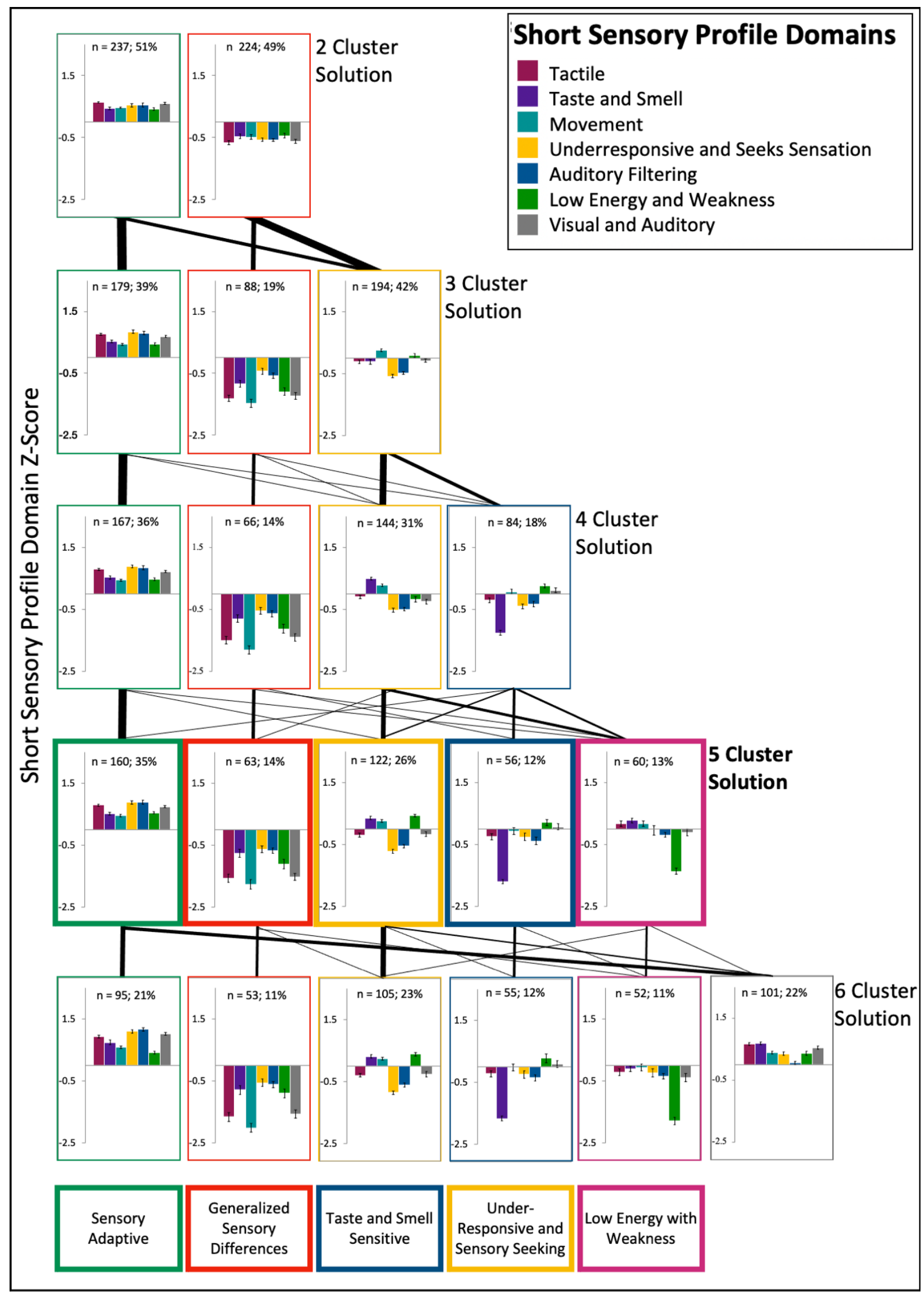


Figure 2: Short Sensory Profile Domain Z-Scores across the k 2 to 6 cluster solutions for the ADHD sample. Negative z-scores are indicative of increased sensory difficulties. Line weights between cluster solutions represent the number of participants remaining/changing clusters across solutions. Error bars indicate standard error of the mean.

One-way ANOVAs were conducted on the SSP subscales scores separately for the autistic children and children with ADHD (z scores: see Figure $1 \&$ 2; raw scores: see Figure 3) using jamovi to determine whether the SSP subscale scores differed across the 5 sensory phenotypes. For both the ASD and ADHD samples, all 7 subscales, tactile, taste/smell, movement, underresponsive/sensory seeking, auditory filtering, low energy/weak, and auditory filtering differed meaningfully across the 5 phenotypes (see Figure 1-3; Supplementary Materials B contains the full statistical analyses, while Supplementary Materials C contains correlations between all experimental variables). Internal consistency of the SSP was assessed for each sample, with Cronbach's $\alpha=.923$ and $\alpha=.930$ for the ASD and ADHD samples, respectively, indicating excellent levels of internal consistency (see Supplementary Materials B for subscale $\alpha s)$.

Given the high degree of similarities across the diagnostic groups, a mixed-model ANOVA was conducted using jamovi to examine the interaction between the 7 SSP subscales, 5 sensory phenotypes, and 2 diagnostic groups to determine whether the pattern of sensory processing differed across sensory phenotypes in a different manner for autistic people and people with ADHD. The three-way interaction between SSP subscales, sensory phenotypes, and diagnosis did not exceed our a priori effect size, $\mathrm{F}(24,5676)=12.82, \mathrm{p}<.001, \eta_{\mathrm{p}}{ }^{2}=.051$, thus 
the differences across the diagnostic groups were not considered meaningful (see Supplementary Materials D for full analysis). 


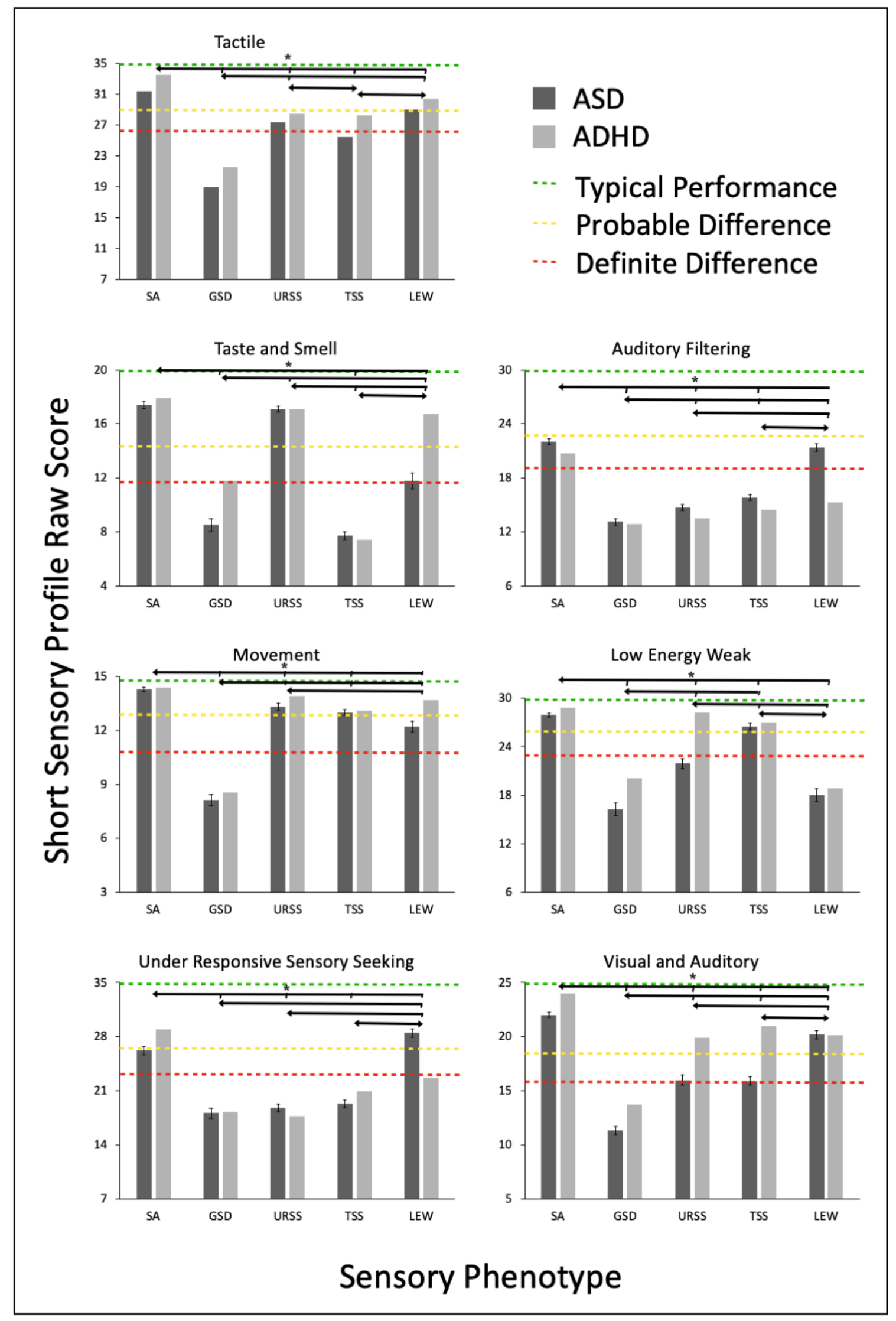


Figure 3: Short Sensory Profile Domain Raw Scores across the five sensory phenotypes: Sensory Adaptive (SA), Generalized Sensory Difference (GSD), Taste and Smell Sensitive (TSS), Under Responsive and Sensory Seeking (URSS), and Low Energy with Weakness for the ASD (black) and ADHD (grey) samples. Error bars indicate standard error of the mean. Dashed lines in green (typical difference), yellow (probable difference), and red (definite difference), classify sensory processing abilities relative to the performance of children without disabilities (McIntosh et al., 1999).

Sensory Phenotypes and Demographic Factors

Age

A two way ANOVA indicated that age did not show meaningful differences across the sensory phenotypes $\left(\mathrm{F}(4,946)=11.64, p<.001, \mathrm{np}^{2}=.047\right)$, diagnostic groups, $(\mathrm{F}(1,946)=$ $\left.0.003, p=.957, \eta_{\mathrm{p}}^{2}<.001\right)$, or the interaction between sensory phenotype and diagnostic group, $(\mathrm{F}(4,946)=9.34, p<.001, \mathrm{np} 2=.038 ;$ see Figure 4a, Table 2$)$

\section{IQ}

A two way ANOVA indicated that full-scale IQ differed across the diagnostic groups, $\left(\mathrm{F}(1,713)=69.21, p<.001, \mathrm{np}^{2}=.088\right)$, but not the sensory phenotypes, $(\mathrm{F}(4,713)=1.29, p$ $=.271, \mathrm{np}^{2}=.007$, or the interaction between diagnosis and sensory phenotype, $(\mathrm{F}(4,713)=$ $3.07, p=.016, \mathrm{np} 2=.017)$. Overall, the people with ADHD had higher full-scale IQs $(\mathrm{M}=99.2$, $\mathrm{SE}=0.93)$, than the autistic people $(\mathrm{M}=83.7, \mathrm{SE}=1.29$; see Table 1; Figure $4 \mathrm{~b})$.

A two way ANOVA indicated that verbal IQ differed across the diagnostic groups, $(\mathrm{F}(1$, $\left.698)=46.30, p<.001, \mathrm{np}^{2}=.062\right)$, but not the sensory phenotypes, $(\mathrm{F}(4,698)=1.43, p=.223$, 
$\mathrm{np}^{2}=.008$, or the interaction between diagnosis and sensory phenotypes, $(\mathrm{F}(4,698)=2.37, p$ $=.051, \mathrm{np} 2=.013)$. Overall, the people with ADHD had higher verbal IQs $(\mathrm{M}=98.7, \mathrm{SE}=$ $0.94)$, than the autistic people $(\mathrm{M}=86.3, \mathrm{SE}=1.31)$.

A two way ANOVA indicated that performance IQ differed across the diagnostic groups, $\left(\mathrm{F}(1,717)=68.93, p<.001, \mathrm{np}^{2}=.088\right)$, but not the sensory phenotypes, $(\mathrm{F}(4,717)=0.93, p$ $=.447, \mathrm{np}^{2}=.005$, or the interaction between diagnosis and sensory phenotype, $(\mathrm{F}(4,717)=$ $0.801, p=.525, \mathrm{np} 2=.004)$. Overall, the people with ADHD had higher performance IQs $(\mathrm{M}=$ 102.0, $\mathrm{SE}=0.90)$, than the autistic people $(\mathrm{M}=87.1, \mathrm{SE}=1.35)$.

Sex assigned at birth

A test of independence indicated that sex did not vary across the phenotypes for the ASD sample $\left(x^{2}(4)=13.24, p=.010\right.$, Cramer's $\left.V=.164\right)$, or the ADHD sample $\left(x^{2}(4)=1.61, p\right.$ $=.806$, Cramer's $V=.059 ;$ see Figure 4c).
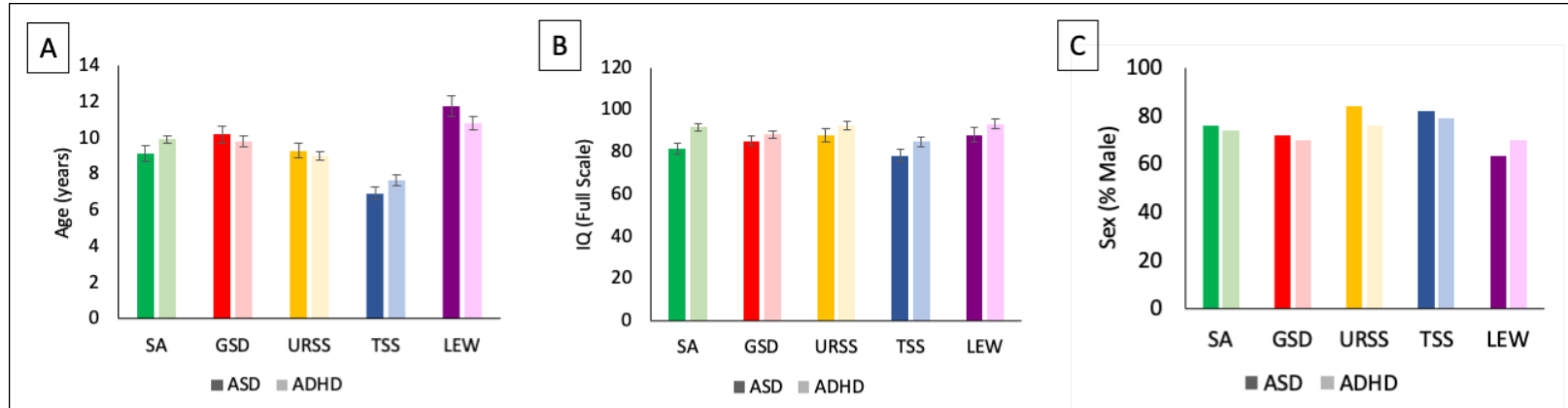

Figure 4: Age (A), IQ (B), and Sex (C) assigned at birth as a function of Sensory Phenotype (Sensory phenotypes: Sensory Adaptive (SA), Generalized Sensory Difference (GSD), Taste and Smell Sensitive (TSS), Under Responsive and Sensory Seeking (URSS), and Low Energy with Weakness) and Diagnostic Group (ASD - solid, ADHD - shaded). Error bars indicate standard error of the mean. 
Table 2: Descriptive and Test Statistics for Measured Variables

\begin{tabular}{|c|c|c|c|c|c|c|}
\hline & & $\begin{array}{c}\text { SA } \\
M(S D) \\
\end{array}$ & $\begin{array}{c}\text { GSD } \\
M(S D)\end{array}$ & $\begin{array}{c}\text { URSS } \\
M \text { (SD) }\end{array}$ & $\begin{array}{c}\text { TSS } \\
\mathrm{M}(\mathrm{SD}) \\
\end{array}$ & $\begin{array}{c}\text { LEW } \\
M(S D) \\
\end{array}$ \\
\hline \multirow[t]{2}{*}{ Age } & ASD & $9.13(4.77)$ & $10.18(4.17)$ & $9.28(3.96)$ & $6.93(3.72)$ & $11.77(4.81)$ \\
\hline & ADHD & $9.90(3.80)$ & $9.77(3.64)$ & $9.00(3.33)$ & $7.64(3.60)$ & $10.79(4.18)$ \\
\hline \multicolumn{7}{|l|}{ IQ } \\
\hline \multirow[t]{2}{*}{ Full-Scale } & ASD & $81.36(27.06)$ & $85.10(20.45)$ & 87.71 (29.09) & 78.19 (26.09) & $88.02(26.08)$ \\
\hline & ADHD & $91.63(23.90)$ & $88.20(20.18)$ & $92.41(24.74)$ & $84.73(25.67)$ & $93.17(23.04)$ \\
\hline \multirow[t]{2}{*}{ Verbal } & ASD & $83.85(25.50)$ & $87.26(21.25)$ & $88.39(27.27)$ & $82.51(24.85)$ & $90.42(25.27)$ \\
\hline & ADHD & 93.95 (22.09) & 88.89 (20.99) & $93.69(23.46)$ & $88.65(23.70)$ & $94.50(21.92)$ \\
\hline \multirow[t]{2}{*}{ Performance } & ASD & $86.76(28.21)$ & $85.94(20.92)$ & $88.57(30.11)$ & $85.59(26.14)$ & $88.48(24.61)$ \\
\hline & ADHD & $96.67(23.31)$ & 90.61 (21.99) & $94.25(24.80)$ & $91.78(24.48)$ & $94.80(22.70)$ \\
\hline Sex & ASD & $30 F, 97 M$ & $22 \mathrm{~F}, 56 \mathrm{M}$ & $17 F, 89 M$ & $20 \mathrm{~F}, 93 \mathrm{M}$ & $26 \mathrm{~F}, 45 \mathrm{M}$ \\
\hline \multirow[t]{2}{*}{ RBS-R Total Score } & ASD & $17.69(15.24)$ & $33.49(22.86)$ & $29.76(19.56)$ & $30.28(18.92)$ & $25.25(21.24)$ \\
\hline & ADHD & $12.87(13.84)$ & $28.10(22.19)$ & $23.89(18.14)$ & $26.88(18.64)$ & $19.72(19.13)$ \\
\hline \multirow[t]{2}{*}{ Self Injury } & ASD & $1.29(2.10)$ & $3.56(4.13)$ & $3.23(3.90)$ & $2.44(2.43)$ & $3.11(3.76)$ \\
\hline & ADHD & $1.15(2.09)$ & $2.94(3.76)$ & $2.68(3.64)$ & 2.19 (2.39) & $2.17(3.18)$ \\
\hline \multirow[t]{2}{*}{ Stereotypy } & ASD & $4.28(3.74)$ & $6.92(5.33)$ & $7.16(4.88)$ & $6.65(4.53)$ & $5.04(5.35)$ \\
\hline & ADHD & $2.68(3.42)$ & $5.71(5.23)$ & $5.51(4.85)$ & $5.60(4.43)$ & $4.07(4.91)$ \\
\hline \multirow[t]{2}{*}{ Compulsions } & ASD & 2.58 (3.49) & $5.21(5.54)$ & $4.30(4.42)$ & $4.50(4.33)$ & $3.18(3.85)$ \\
\hline & ADHD & $1.89(3.05)$ & $4.33(4.97)$ & $3.14(3.83)$ & $3.77(4.11)$ & $2.49(3.49)$ \\
\hline \multirow{2}{*}{$\begin{array}{l}\text { Ritualistic/ } \\
\text { Sameness }\end{array}$} & ASD & $8.63(8.51)$ & $15.78(9.98)$ & $13.34(9.51)$ & $14.96(9.75)$ & $12.20(10.07)$ \\
\hline & ADHD & $6.37(7.45)$ & 13.28 (9.99) & $10.93(8.54)$ & 13.55 (9.63) & $9.54(9.11)$ \\
\hline
\end{tabular}

\begin{tabular}{lcccccc}
\hline SCQ & ASD & $15.83(6.56)$ & $23.14(7.40)$ & $20.83(7.08)$ & $20.98(6.63)$ & $17.89(7.02)$ \\
& ADHD & $9.20(7.83)$ & $17.81(9.50)$ & $14.47(8.43)$ & $16.40(8.74)$ & $12.41(8.53)$ \\
SWAN & & & & & & \\
$\quad$ Inattention & ASD & $3.42(2.85)$ & $5.03(2.86)$ & $5.10(2.86)$ & $4.28(3.11)$ & $3.26(3.11)$ \\
& ADHD & $4.47(3.10)$ & $5.58(2.75)$ & $5.73(2.73)$ & $4.80(3.12)$ & $4.39(3.15)$ \\
Hyperactivity & ASD & $2.57(2.76)$ & $4.08(3.08)$ & $4.62(3.26)$ & $4.28(3.21)$ & $2.13(2.72)$ \\
& ADHD & $3.07(2.96)$ & $4.73(3.15)$ & $5.03(3.08)$ & $4.35(3.28)$ & $2.97(3.06)$ \\
\hline
\end{tabular}

Note: Sensory Adaptive (SA), Generalized Sensory Differences (GSD), Taste and Smell Sensitivity (TSS), Under-Responsive Sensory Seeking (URSS), Movement Difficulties with Low Energy and Weakness (M/LEW), Intelligence Quotient (IQ), Repetitive Behaviours Scale Revised (RBS-R), Social Communication Questionnaire (SCQ), Strengths and Weakness of Attention-Deficit/Hyperactivity Disorder Symptoms of Normal Behaviour Scale (SWAN). Contrasts are significant at $p<.05$.

Sensory Phenotypes and Neurodevelopmental Disorder Traits

ASD Traits 
Autistic social behaviours, measured by the SCQ, were also found to differ across the diagnostic groups, $\left(\mathrm{F}(1,867)=702.830, p<.001, \mathrm{np}^{2}=.448\right)$, and sensory phenotypes, $(\mathrm{F}(4$, $\left.867)=37.16, p<.001, \mathrm{np}^{2}=.146\right)$, but the interaction was not statistically meaningful, $(\mathrm{F}(4$, $867)=0.15, p=.964, \mathrm{np}^{2}=.001$; see Figure 5; Table 2). In addition, the internal consistency of the SCQ was assessed, with Cronbach's $\alpha=.920$, indicating excellent internal consistency.

Restricted and repetitive behaviours, indexed by RBS-R total scores, differed across the diagnostic groups, $\left(\mathrm{F}(1,946)=84.14, p<.001, \mathrm{np}^{2}=.082\right)$, and sensory phenotypes, $(\mathrm{F}(4,946)$ $\left.=23.53, p<.001, \mathrm{np}^{2}=.090\right)$, but the interaction between diagnostic groups and sensory phenotypes, $\left(\mathrm{F}(4,946)=0.36, p=.834, \mathrm{np}^{2}=.002\right)$, was not statistically meaningful (see Figure 4; Table 2).

Two-way ANOVAs were also conducted on the stereotypy, self-injury, compulsions, and ritualistic-sameness subscales of the RBS-R. Stereotypy and ritualistic-sameness differed across the diagnostic groups, $\left(\mathrm{F}(1,946)=91.68, p<.001, \mathrm{np}^{2}=.088\right)$ and $(\mathrm{F}(1,946)=69.58, p<.001$, $\left.\mathrm{np}^{2}=.069\right)$, respectively, and the sensory phenotypes, $\left(\mathrm{F}(4,946)=18.08, p<.001, \mathrm{np}^{2}=.071\right)$ and $\left(\mathrm{F}(4,946)=21.16, p<.001, \mathrm{np}^{2}=.082\right)$, respectively. All other comparisons were not statistically meaningful (see Figure 5; Table 2). In addition, the internal consistency of the RBSR was assessed, with Cronbach's $\alpha=.942$, indicating excellent internal consistency. 


\section{Social Behaviours}

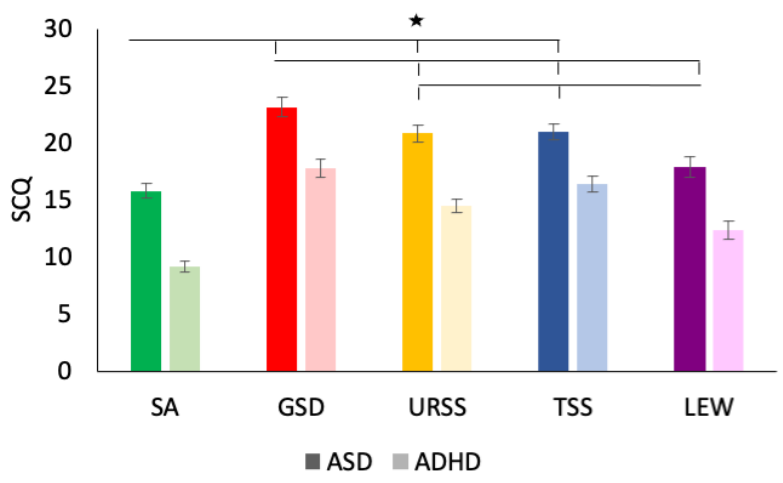

Restricted \& Repetitive Behaviours

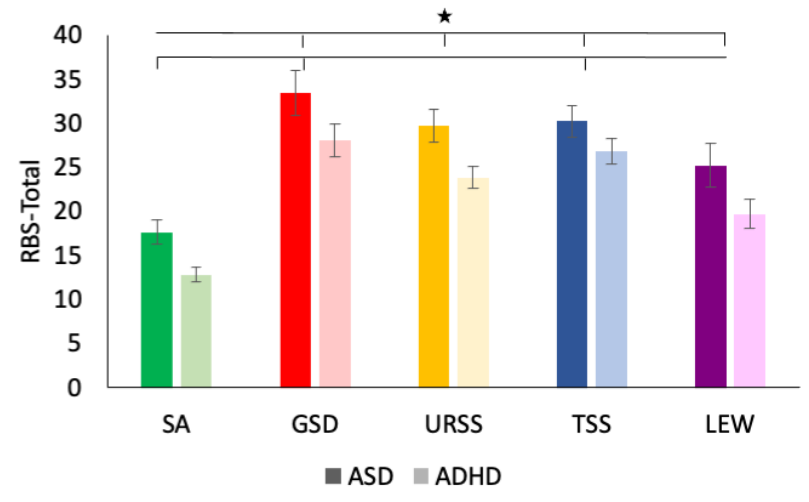

\section{Restricted \& Repetitive Behaviours Subscales}
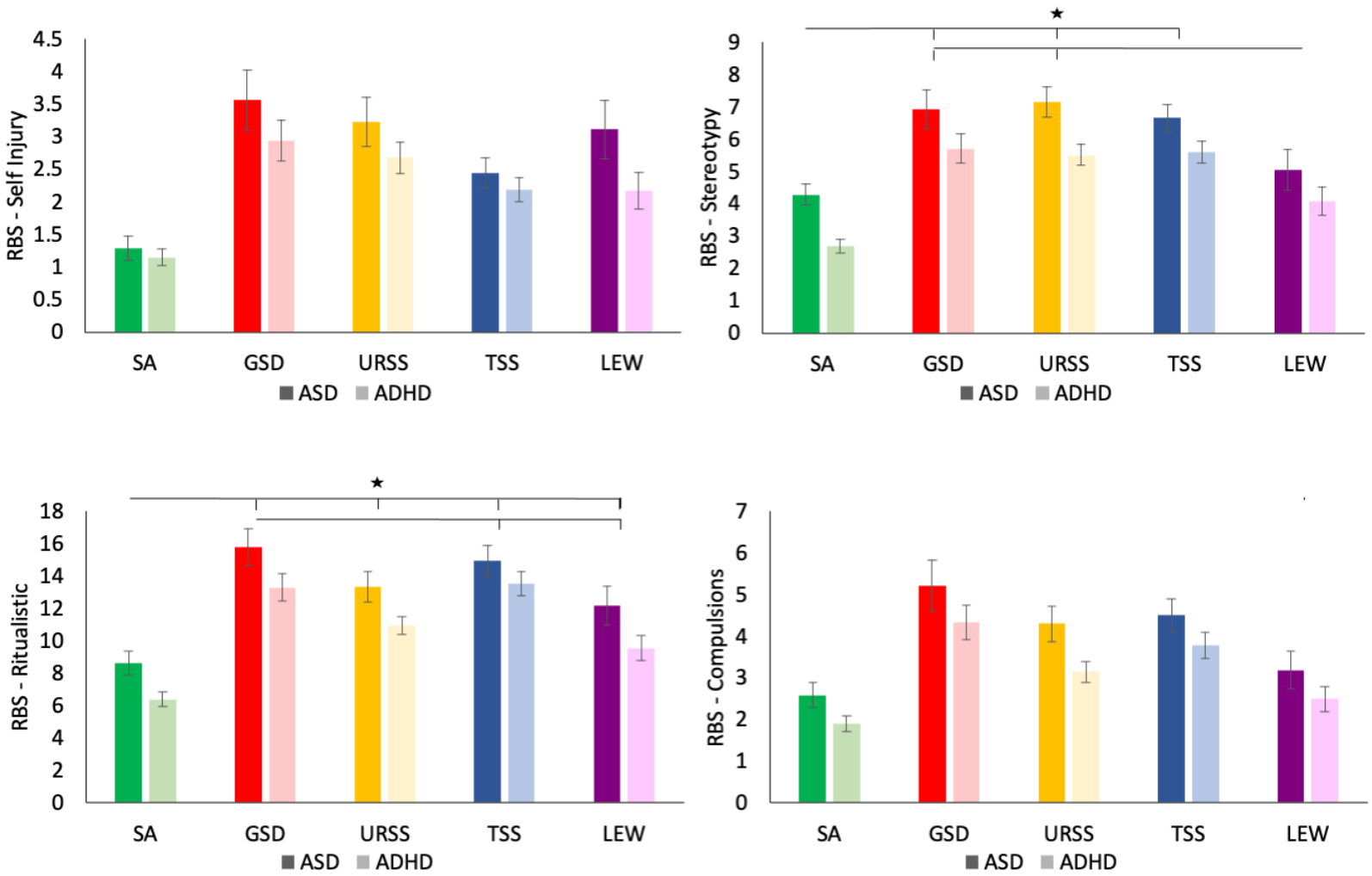

Figure 5: Social Behaviours, measured by the SCQ, and Repetitive Behaviours, measured by the RBS-R, and as a function of sensory phenotype. Error bars indicate standard error of the mean.

Higher scores are indicative of more social difficulties on the SCQ, and more repetitive behaviours on the RBS-R. Note: * indicates significance at $p<.05$. 


\section{ADHD Traits}

SWAN scores indicated that inattention scores did not meaningfully differ across diagnosis, sensory phenotypes, or their interaction. However, hyperactivity scores differed across sensory phenotypes, $\left(\mathrm{F}(1,802)=17.05, p<.001, \mathrm{np}^{2}=.078\right.$; see Figure 6; Table 2). In addition, the internal consistency of the SWAN was assessed, with Cronbach's $\alpha=.939$, indicating excellent internal consistency.

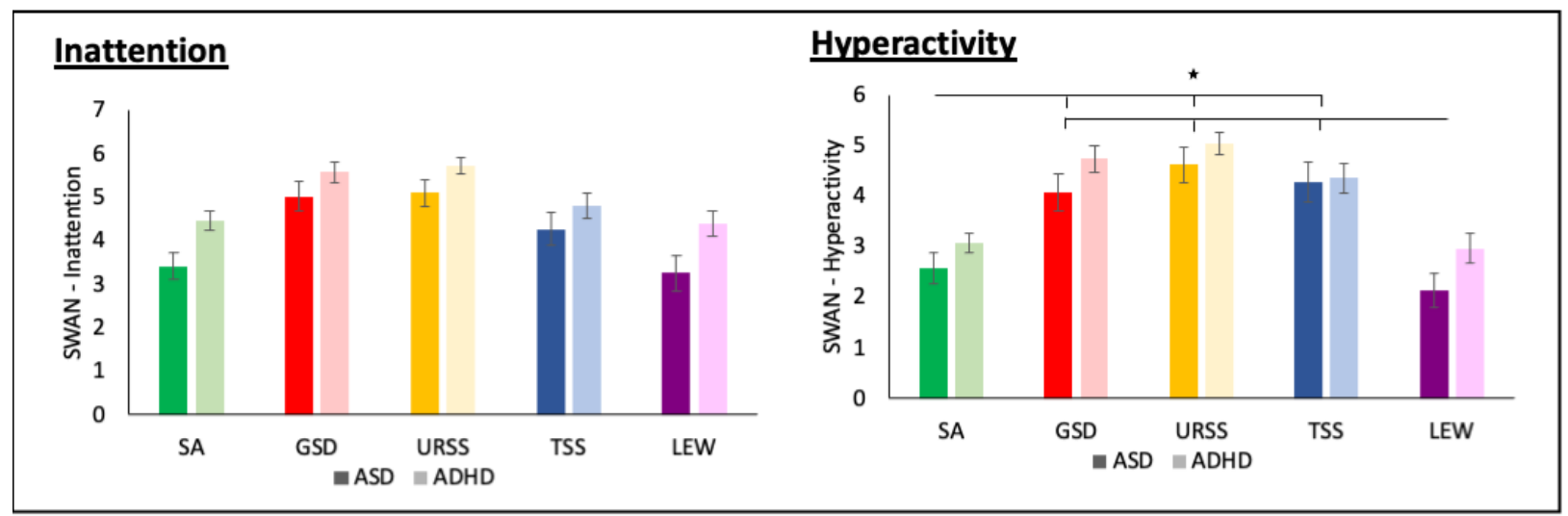

Figure 6: ADHD traits (Inattention, Hyperactivity) as measured by the SWAN, as a function of Sensory Phenotype. Higher scores on the SWAN are indicative of more ADHD traits. Error bars indicate standard error of the mean. Note: * indicates significance at $\mathrm{p}<.05$.

\section{Combined Model}

Given the similarities in sensory processing patterns across autistic people and people with ADHD in this sample, a k-means cluster analysis was conducted on the combined sample to compare the distribution of autistic people and people with ADHD across the five sensory phenotypes (see Figure 6). For the autistic sample, the GSD phenotype characterized the sensory processing abilities of the fewest number of people (17\%), while the largest amount of the 
sample was characterized by the SA phenotype (23\%). Similarly, for the ADHD sample, the GSD A test of independence indicated that the distribution of autistic people and people with ADHD across the fives sensory phenotypes was not meaningfully different, $\left(x^{2}(4)=70.4, p\right.$ $<.001$, Cramer's $V=.271)$; see Figure 6 .

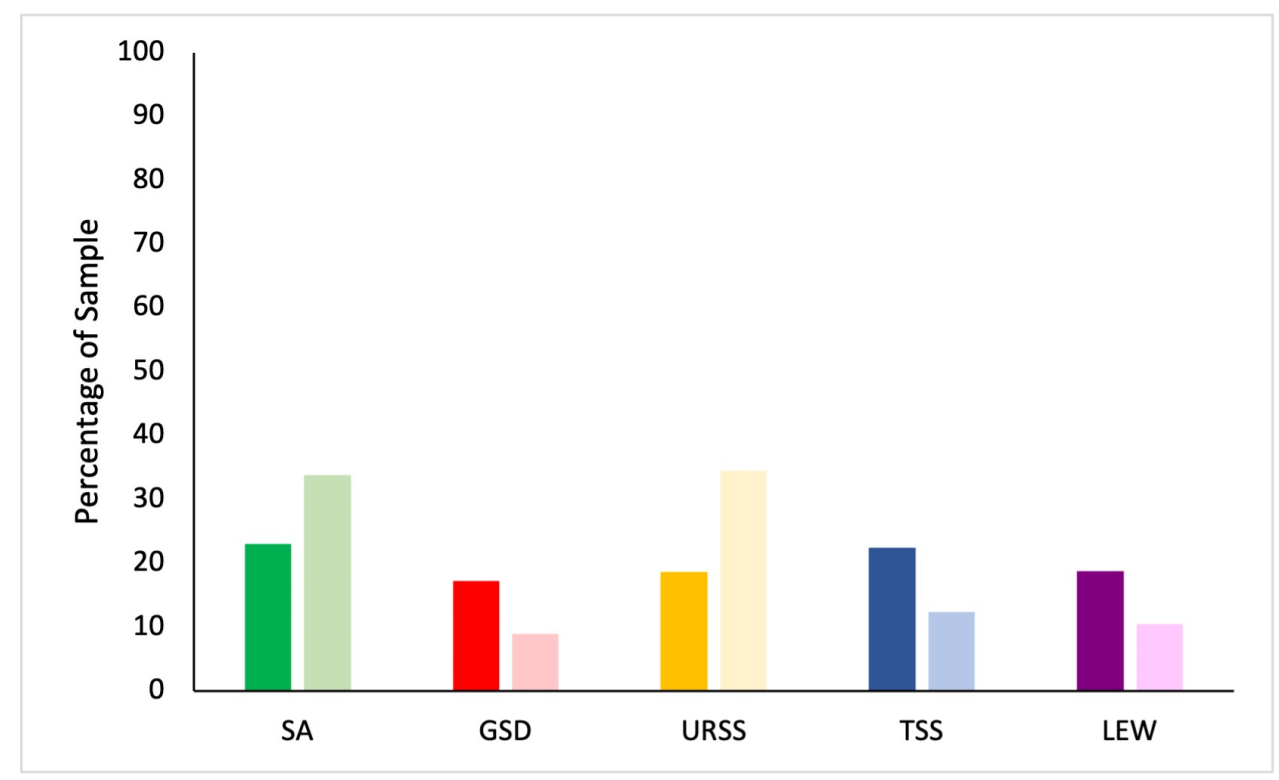

Figure 7: Percentage of the ASD and ADHD samples characterized by each sensory phenotype $(\mathrm{SA}=$ sensory adaptive, $\mathrm{GSD}=$ generalized sensory difference, $\mathrm{URSS}=$ underresponsive sensory seeking, TSS = taste and smell sensitive, LEW = low energy with weakness).

\section{Discussion}

The transdiagnostic nature of sensory phenotypes

The primary aim of the current study was to assess whether data-driven sensory phenotypes originally observed in autism are transdiagnostic in nature. Five sensory phenotypes independently emerged in each diagnostic group that did not meaningfully differ across the diagnostic groups, suggesting sensory processing abilities are in fact transdiagnostic in nature. 
Further, the patterns of clinical traits across the sensory phenotypes were similar for autistic people and people with ADHD. Finally, when clustered as a single, transdiagnostic group, people from both diagnostic groups were broadly distributed across phenotypes, suggesting that diagnostic category was not able to reliably predict sensory characteristics. Thus, this research highlights the transdiagnostic nature of sensory processing in ASD and ADHD and suggests that observed differences in sensory processing across diagnoses may be quantitative, rather than qualitative in nature. Further, it suggests that sensory processing in these groups involves a shared underlying neurobiological mechanism and argues for similar practices for studying and supporting sensory processing abilities in these neurodevelopmental conditions.

This research complements a growing body of literature that reveals that the existing diagnostic labels of ASD and ADHD demonstrate poor explanatory power (Baribeau et al., 2015, 2019; Jacobs et al., 2021; Krakowski et al., 2020; Kuskhi et al., 2019, 2021). For example, when considering cognitive features, overlapping cognitive deficits across ASD and ADHD have been identified using latent class analyses (van der Meer et al., 2012). Similar patterns of social perception abilities (Baribeau et al., 2015) and similar social cognitive profiles (Demopoulos et al., 2013) have also been reported across autistic people and people with ADHD, while structural neuroimaging has revealed similarities in the biological substrates of social communication abilities in these same diagnostic groups (Baribeau et al., 2019). Additional structural similarities have been identified across ASD and ADHD by comparing white matter disruption (Ameis et al., 2016) and cortical thickness (Jacobs et al., 2021). Further, twin studies (Ronald et al., 2008) and studies of rare copy number variants (Lionel et al, 2011, 2014) highlight overlapping etiological contributions to ASD and ADHD. In light of emerging evidence that the ASD and ADHD diagnostic categories may not correspond with distinct conditions, focusing on data-driven 
profiles of autistic people and people with ADHD, including their sensory abilities, rather than their categorical diagnoses, could allow for more focused interventions that target the sensory domains and behaviours that create the greatest challenges for these people (Genovese \& Butler, 2020; Jeste et al., 2014; Scheerer et al., 2021). Given these sensory phenotypes highlight both sensory abilities and difficulties, this approach may facilitate the development of environments that capitalize on sensory abilities, while also supporting sensory difficulties.

\section{Sensory phenotype characteristics}

Data-driven phenotypes have been previously used to characterize the sensory abilities of autistic people (Dwyer et al., 2020; Lane et al., 2010, 2011, 2014; Scheerer et al., 2021; Uljarevic et al., 2016). The current results extend these findings by demonstrating that the sensory processing abilities of people with ADHD can also be clustered into five discrete sensory phenotypes. In both the sample of autistic people and people with ADHD, the first two phenotypes to emerge were a sensory adaptive (SA) phenotype and a generalized sensory difference (GSD) phenotype. People characterized by the SA phenotype were reported by their parents to have typical sensory processing abilities across all sensory domains, except for the auditory filtering domain where they showed probable differences relative to normative data from a non-clinical population (McIntosh et al., 1999). People characterized by the GSD phenotype were described by their parents as having definite differences in sensory processing abilities across all seven sensory domains. Thus, the SA and GSD phenotypes represent the opposite extremes of the spectrum of sensory processing abilities and highlight the fact that autistic people and people with ADHD show a broad range of sensory processing abilities. Three additional sensory phenotypes were identified that demonstrated intermediate patterns of sensory 
processing abilities, with some typical, probable, and definite sensory processing difference patterns.

The first of the intermediate phenotypes to emerge was the under-responsive/sensory seeking (URSS) phenotype. The URSS phenotype characterized people who were reported to have definite differences in under responsivity and sensory seeking as well as auditory filtering, with probable differences in low energy and weakness, visual and auditory processing, and tactile processing. The taste and smell sensitivity (TSS) phenotype characterized people who were described as having definite differences in taste and smell processing, under responsivity and sensory seeking, and auditory filtering, while they were reported to have probable differences with tactile and visual and auditory processing. Lastly, people characterized by the low energy weakness (LEW) phenotype were described as having difficulties with low energy and weakness, auditory filtering, and tactile processing. They were also reported to have probable differences with taste and smell processing, movement, and under responsivity and sensory seeking behaviours. While the URSS (Lane et al., 2011; Scheerer et al., 2021), TSS (Lane et al., 2010, 2011, 2014; Scheerer et al., 2021), and LEW (Lane et al., 2014; Scheerer et al., 2021) phenotypes have been previously used to characterize the sensory abilities of autistic people, the current results extend these findings by demonstrating that these same phenotypes can characterize sensory processing abilities of people with ADHD. Given the similarities in the way that the sensory processing abilities clustered across the autistic people and people with ADHD in this study, these results suggest that ASD and ADHD diagnoses may not be associated with unique patterns of sensory processing abilities. Relating sensory phenotypes and clinically relevant characteristics 
The sensory processing abilities of autistic people have previously been associated with core ASD features (i.e. restricted and repetitive behaviours and social communication difficulties; Lane et al., 2010; Scheerer et al., 2021; Tillmann et al., 2020) and ADHD traits (i.e., inattention and hyperactivity; Scheerer et al., 2021; Tillmann et al., 2020). Accordingly, both features of ASD and ADHD were found to differ across the five sensory phenotypes characterizing the sample of autistic people. Further, a similar relationship between ASD and ADHD features and sensory phenotypes was also identified in the ADHD sample. Specifically, when considering restricted and repetitive behaviours, the people characterized by the most adaptive sensory processing phenotype (the SA phenotype), were reported to demonstrate the lowest levels of stereotypy and ritualistic behaviours. Similarly, stereotypy was also reported to be lower in people characterized by the LEW phenotype, relative to those characterized by the GSD, and URSS phenotypes. People who demonstrated the highest level of sensory processing difficulties, those characterized by the GSD phenotype, showed the most ritualistic behaviours. These findings are aligned with previous work that reported increased repetitive behaviours in autistic people with more sensory challenges (Tillmann et al., 2020), but highlight a similar association in people with ADHD. A similar pattern emerged for social communication abilities, as people characterized by the SA and LEW phenotypes were reported to have the best social communication abilities, while those characterized by the GSD phenotype were reported to have the most difficulties with social communication, which is again in line with previous reports of autistic people (Tillmann et al., 2020). While the relationship between sensory processing abilities and autistic traits was similar across the two diagnostic groups, autistic people were reported to have more social communication difficulties and restricted and repetitive behaviours overall. Given the association between sensory abilities and autistic traits was similar across both 
diagnostic groups, this suggests that this difference is quantitative, but not qualitative in nature. When considering the core ADHD traits of inattention and hyperactivity, overall, these traits were not found to differ across the ASD and ADHD samples. In line with previous findings (Krakowski et al., 2020), although the autistic people in this sample did not have a diagnosis of ADHD, their reported levels of inattention and hyperactivity matched those of the people with ADHD. When considering the relationship with sensory processing abilities, inattention was not found to differ across the five sensory phenotypes. However, hyperactivity was higher for those characterized by the GSD, URSS, and TSS phenotypes, relative to those characterized by the SA and LEW phenotypes. Thus, the sensory processing abilities of autistic people and people with ADHD do not appear to show unique patterns of association with the core ASD or ADHD traits.

Given the transdiagnostic nature of sensory processing in ASD and ADHD, future work should investigate whether these same sensory phenotypes can adequately describe sensory processing differences in other populations that demonstrate sensory processing challenges, such as people with OCD (Dar, Kahn, Carmeli, 2012) and neurodevelopmentally typical children (Dunn et al., 2016) and adults (Ben-Avi, Almagor, \& Engel-Yeger, 2012; Pohl, Dunn \& Brown, 2003). Further, while the current work identified relationships between sensory processing abilities and current traits, future work should assess whether these phenotypes are also predictive of future behaviours.

\section{Limitations}

This study is not without limitations. As the results were based on subjective parent reports of sensory processing and traits associated with ASD and ADHD, this limits the generalizability of the findings. Further, while the SSP is widely used to measure sensory processing in autistic people and people with ADHD, there is limited psychometric evidence of 
convergent validity (Williams et al., 2018). In addition, given these data were obtained from a large provincial database, in some instances a subset of the measures were completed for some people.

\section{Conclusions}

These results demonstrate that sensory processing abilities in neurodevelopmental conditions are transdiagnostic in nature. Specifically, sensory processing patterns in autistic people and people with ADHD are highly similar. Further these results suggest that sensory processing abilities in autistic people and people with ADHD can be characterized by sensory phenotypes that not only parse the heterogeneity in sensory processing abilities, but are also associated with the same pattern of clinical differences in ASD and ADHD traits. Thus, these results provide support for the notion that the ASD and ADHD diagnostic categories may not correspond to conditions with distinct underlying mechanisms of dysfunction (Baribeau et al., 2019; Insel et al., 2010; Jacobs et al., 2021; Krakowski et al., 2020; Kushki et al., 2019, 2021).

\section{Author Note}

This research was supported by Nichole Scheerer's BrainsCAN Postdoctoral Fellowship at Western University, funded by the Canada First Research Excellence Fund (CFREF). RS is funded by an NSERC Discovery Grant (RGPIN-2017-04656), a SSHRC Insight Grant (4352017-0936), the University of Western Ontario Faculty Development Research Fund, the province of Ontario Early Researcher Award, and a Canadian Foundation for Innovation John R. Evans Leaders Fund (37497). EK is supported by the Masonic Foundation of Ontario. The POND Network is part of an Integrated Discovery System (IDS) that is funded by the Ontario Brain Institute (OBI). 
We would like to thank all of the families that have participated in the POND Network for supporting this research. We would also like to thank our lab lab(rador) Daisy Doodle Scheerer, the Sensory Perception Research Lab's research support lab(rador).

Correspondence concerning this article should be addressed to Dr. Nichole Scheerer

Western University, Brain and Mind Institute, 1151 Richmond St, London, ON, Canada, N6A

3K7, nikkischeerer@gmail.com.

\section{References}

Ameis, S. H., Lerch, J. P., Taylor, M. J., Lee, W., Viviano, J. D., Pipitone, J., ... \& Anagnostou, E. (2016). A diffusion tensor imaging study in children with ADHD, autism spectrum disorder, OCD, and matched controls: distinct and non-distinct white matter disruption and dimensional brain-behavior relationships. American Journal of Psychiatry, 173(12), 1213-1222.

American Psychiatric Association. (2013). Diagnostic and statistical manual of mental disorders (5th ed.). American Psychiatric Association.

Arnett, A. B., Pennington, B. F., Friend, A., Willcutt, E. G., Byrne, B., Samuelsson, S., \& Olson, R. K. (2013). The SWAN captures variance at the negative and positive ends of the ADHD symptom dimension. Journal of attention disorders, 17(2), 152-162.

Ausderau, K. K., Furlong, M., Sideris, J., Bulluck, J., Little, L. M., Watson, L. R., ... \& Baranek, G. T. (2014). Sensory subtypes in children with autism spectrum disorder: latent profile transition analysis using a national survey of sensory features. Journal of Child Psychology and Psychiatry, 55(8), 935-944.

Baribeau, D. A., Doyle-Thomas, K. A., Dupuis, A., Iaboni, A., Crosbie, J., McGinn, H., ... \& Anagnostou, E. (2015). Examining and comparing social perception abilities across childhoodonset neurodevelopmental disorders. Journal of the American Academy of Child \& Adolescent Psychiatry, 54(6), 479-486

Baribeau, D. A., Dupuis, A., Paton, T. A., Hammill, C., Scherer, S. W., Schachar, R. J., ... \& Anagnostou, E. (2019). Structural neuroimaging correlates of social deficits are similar in autism spectrum disorder and attention-deficit/hyperactivity disorder: analysis from the POND Network. Translational psychiatry, 9(1), 1-14.

Ben-Avi, N., Almagor, M., \& Engel-Yeger, B. (2012). Sensory processing difficulties and interpersonal relationships in adults: an exploratory study. Psychology, 3(01), 70. 
Ben-Sasson, A., Hen, L., Fluss, R., Cermak, S. A., Engel-Yeger, B., \& Gal, E. (2009). A metaanalysis of sensory modulation symptoms in individuals with autism spectrum disorders. Journal of autism and developmental disorders, 39(1), 1-11.

Brierley, N. J., McDonnell, C. G., Parks, K., Schulz, S. E., Dalal, T. C., Kelley, E., ... \& Stevenson, R. A. (2021). Factor Structure of repetitive behaviors across autism spectrum disorder and attention-deficit/hyperactivity disorder. Journal of Autism and Developmental Disorders, 51(10), 3391-3400.

Dar, R., Kahn, D. T., \& Carmeli, R. (2012). The relationship between sensory processing, childhood rituals and obsessive-compulsive symptoms. Journal of behavior therapy and experimental psychiatry, 43(1), 679-684.

DeBoth, K. K., \& Reynolds, S. (2017). A systematic review of sensory-based autism subtypes. Research in autism spectrum disorders, 36, 44-56.

Demopoulos, C., Hopkins, J., \& Davis, A. (2013). A comparison of social cognitive profiles in children with autism spectrum disorders and attention-deficit/hyperactivity disorder: a matter of quantitative but not qualitative difference?. Journal of autism and developmental disorders, 43(5), 1157-1170.

Dunn, W. (2014). Sensory profile 2. Psych Corporation.

Dunn, W., Little, L., Dean, E., Robertson, S., \& Evans, B. (2016). The state of the science on sensory factors and their impact on daily life for children: A scoping review. OTJR: Occupation, Participation and Health, 36(2_suppl), 3S-26S.

Dwyer, P., Saron, C. D., \& Rivera, S. M. (2020). Identification of Longitudinal Sensory Subtypes in Typical Development and Autism Spectrum Development Using Growth Mixture Modelling. Research in Autism Spectrum Disorders, 78, 101645.

Genovese, A., \& Butler, M. G. (2020). Clinical assessment, genetics, and treatment approaches in autism spectrum disorder (ASD). International journal of molecular sciences, 21(13), 4726.

Gotham, K., Risi, S., Pickles, A., \& Lord, C. (2007). The Autism Diagnostic Observation Schedule: revised algorithms for improved diagnostic validity. Journal of autism and developmental disorders, 37(4), 613-627.

Hartigan, J. A., \& Wong, M. A. (1979). Algorithm AS 136: A k-means clustering algorithm. Journal of the royal statistical society. series c (applied statistics), 28(1), 100-108.

Hossain, M. M., Khan, N., Sultana, A., Ma, P., McKyer, E. L. J., Ahmed, H. U., \& Purohit, N. (2020). Prevalence of comorbid psychiatric disorders among people with autism spectrum 
disorder: An umbrella review of systematic reviews and meta-analyses. Psychiatry research, 287, 112922.

Insel, T., Cuthbert, B., Garvey, M., Heinssen, R., Pine, D. S., Quinn, K., ... \& Wang, P. (2010). Research domain criteria (RDoC): toward a new classification framework for research on mental disorders.

Jacobs, G. R., Voineskos, A. N., Hawco, C., Stefanik, L., Forde, N. J., Dickie, E. W., ... \& Ameis, S. H. (2021). Integration of brain and behavior measures for identification of data-driven groups cutting across children with ASD, ADHD, or OCD. Neuropsychopharmacology, 46(3), 643-653.

Jeste, S. S., \& Geschwind, D. H. (2014). Disentangling the heterogeneity of autism spectrum disorder through genetic findings. Nature Reviews Neurology, 10(2), 74-81.

Krakowski, A. D., Cost, K. T., Anagnostou, E., Lai, M. C., Crosbie, J., Schachar, R., ... \& Szatmari, P. (2020). Inattention and hyperactive/impulsive component scores do not differentiate between autism spectrum disorder and attention-deficit/hyperactivity disorder in a clinical sample. Molecular autism, 11(1), 1-13.

Kushki, A., Anagnostou, E., Hammill, C., Duez, P., Brian, J., Iaboni, A., ... \& Lerch, J. P. (2019). Examining overlap and homogeneity in ASD, ADHD, and OCD: a data-driven, diagnosis-agnostic approach. Translational psychiatry, 9(1), 1-11.

Kushki, A., Cardy, R. E., Panahandeh, S., Malihi, M., Hammill, C., Brian, J., ... \& Anagnostou, E. (2021). Cross-Diagnosis Structural Correlates of Autistic-Like Social Communication Differences. Cerebral Cortex.

Lai, M. C., Kassee, C., Besney, R., Bonato, S., Hull, L., Mandy, W., ... \& Ameis, S. H. (2019). Prevalence of co-occurring mental health diagnoses in the autism population: a systematic review and meta-analysis. The Lancet Psychiatry, 6(10), 819-829.

Lam, K. S., \& Aman, M. G. (2007). The Repetitive Behavior Scale-Revised: independent validation in individuals with autism spectrum disorders. Journal of autism and developmental disorders, 37(5), 855-866.

Lane, A. E., Young, R. L., Baker, A. E., \& Angley, M. T. (2010). Sensory processing subtypes in autism: Association with adaptive behavior. Journal of autism and developmental disorders, 40(1), 112-122.

Lane, A. E., Dennis, S. J., \& Geraghty, M. E. (2011). Brief report: further evidence of sensory subtypes in autism. Journal of autism and developmental disorders, 41(6), 826-831.

Lane, A. E., Molloy, C. A., \& Bishop, S. L. (2014). Classification of Children With Autism Spectrum Disorder by Sensory Subtype: A Case for Sensory-Based Phenotypes. Autism Research, 7(3), 322-333. 
Lionel, A. C., Crosbie, J., Barbosa, N., Goodale, T., Thiruvahindrapuram, B., Rickaby, J., ... \& Scherer, S. W. (2011). Rare copy number variation discovery and cross-disorder comparisons identify risk genes for ADHD. Science translational medicine, 3(95), 95ra75-95ra75.

Lionel, A. C., Tammimies, K., Vaags, A. K., Rosenfeld, J. A., Ahn, J. W., Merico, D., ... \& Scherer, S. W. (2014). Disruption of the ASTN2/TRIM32 locus at 9q33. 1 is a risk factor in males for autism spectrum disorders, ADHD and other neurodevelopmental phenotypes. Human molecular genetics, 23(10), 2752-2768.

Liss, M., Saulnier, C., Fein, D., \& Kinsbourne, M. (2006). Sensory and attention abnormalities in autistic spectrum disorders. Autism, 10(2), 155-172.

Little, L. M., Dean, E., Tomchek, S. D., \& Dunn, W. (2017). Classifying sensory profiles of children in the general population. Child: care, health and development, 43(1), 81-88.

Little, L. M., Dean, E., Tomchek, S., \& Dunn, W. (2018). Sensory processing patterns in autism, attention deficit hyperactivity disorder, and typical development. Physical \& occupational therapy in pediatrics, 38(3), 243-254.

Lord, C., Rutter, M., \& Le Couteur, A. (1994). Autism Diagnostic Interview-Revised: a revised version of a diagnostic interview for caregivers of individuals with possible pervasive developmental disorders. Journal of autism and developmental disorders, 24(5), 659-685.

Mangeot, S. D., Miller, L. J., McIntosh, D. N., McGrath-Clarke, J., Simon, J., Hagerman, R. J., \& Goldson, E. (2001). Sensory modulation dysfunction in children with attention-deficithyperactivity disorder. Developmental medicine and child neurology, 43(6), 399-406.

McIntosh, D. N., Miller, L. J., Shyu, V., \& Dunn, W. (1999). Development and validation of the short sensory profile. Sensory profile manual, 59-73.

Mullen, E. M. (1995). Mullen scales of early learning (pp. 58-64). Circle Pines, MN: AGS.

Parks, K., Schulz, S., McDonnell, C. G., Anagnostou, E., Nicolson, R., Kelley, E., ... \& Stevenson, R. (2020). Sensory Processing in ASD and ADHD: A Confirmatory Factor Analysis. PsyArXiv.

Pfeiffer, B., Daly, B. P., Nicholls, E. G., \& Gullo, D. F. (2015). Assessing sensory processing problems in children with and without attention deficit hyperactivity disorder. Physical \& occupational therapy in pediatrics, 35(1), 1-12.

Pohl, P. S., Dunn, W., \& Brown, C. (2003). The role of sensory processing in the everyday lives of older adults. OTJR: Occupation, Participation and Health, 23(3), 99-106.

Roid, G. H., \& Pomplun, M. (2012). The stanford-binet intelligence scales. The Guilford Press. 
Roid, G. H., Miller, L. J., \& Koch, C. (2013). Leiter international performance scale (pp. 1-1). Wood Dale, IL: Stoelting.

Ronald, A., Simonoff, E., Kuntsi, J., Asherson, P., \& Plomin, R. (2008). Evidence for overlapping genetic influences on autistic and ADHD behaviours in a community twin sample. Journal of Child psychology and Psychiatry, 49(5), 535-542.

Rutter, M., Bailey, A., \& Lord, C. (2003). SCQ. The Social Communication Questionnaire. Torrance, CA: Western Psychological Services.

Scheerer, N., Curcin, K., Stojanoski, B., Anagnostou, E., Nicolson, R., Kelley, E., ... \& Stevenson, R. (2021). Exploring Sensory Phenotypes in Autism Spectrum Disorder. Molecular Autism,

Simpson, K., Adams, D., Alston-Knox, C., Heussler, H. S., \& Keen, D. (2019). Exploring the sensory profiles of children on the autism spectrum using the short sensory profile-2 (SSP2). Journal of Autism and Developmental Disorders, 49(5), 2069-2079.

Tillmann, J., Uljarevic, M., Crawley, D., Dumas, G., Loth, E., Murphy, D., ... \& Charman, T. (2020). Dissecting the phenotypic heterogeneity in sensory features in autism spectrum disorder: a factor mixture modelling approach. Molecular autism, 11(1), 1-15.

Tomchek, S. D., \& Dunn, W. (2007). Sensory processing in children with and without autism: a comparative study using the short sensory profile. American Journal of occupational therapy, 61(2), 190-200.

Tomchek, S. D., Huebner, R. A., \& Dunn, W. (2014). Patterns of sensory processing in children with an autism spectrum disorder. Research in Autism Spectrum Disorders, 8(9), 1214-1224.

Uljarević, M., Lane, A., Kelly, A., \& Leekam, S. (2016). Sensory subtypes and anxiety in older children and adolescents with autism spectrum disorder. Autism Research, 9(10), 1073-1078.

Van Der Meer, J. M., Oerlemans, A. M., Van Steijn, D. J., Lappenschaar, M. G., De Sonneville, L. M., Buitelaar, J. K., \& Rommelse, N. N. (2012). Are autism spectrum disorder and attentiondeficit/hyperactivity disorder different manifestations of one overarching disorder? Cognitive and symptom evidence from a clinical and population-based sample. Journal of the American Academy of Child \& Adolescent Psychiatry, 51(11), 1160-1172.

Wechsler, D. (1999). Wechsler Abbreviated Scale of Intelligence (WASI). San Antonio, TX: Psychological Corporation.

Wechsler, D. (2003). Wechsler Intelligence Scale for Children-Fourth Edition (WISC-IV). San Antonio, TX: NCS Pearson. 
Wechsler, D. (2011). Wechsler Abbreviated Scale of Intelligence-Second Edition (WASIII). San Antonio, TX: NCS Pearson.

Wechsler, D. (2012). Technical and interpretative manual: WPPSI-IV. NY: Pearson Inc.

Wechsler, D. (2014). Wechsler Intelligence Scale for Children-Fifth Edition (WISC-V). San Antonio, TX: NCS Pearson.

Williams, Z. J., Failla, M. D., Gotham, K. O., Woynaroski, T. G., \& Cascio, C. (2018).

Psychometric evaluation of the short sensory profile in youth with autism spectrum disorder. Journal of autism and developmental disorders, 48(12), 4231-4249.

Zhong, S., \& Ghosh, J. (2003). A unified framework for model-based clustering. The Journal of Machine Learning Research, 4, 1001-1037. 